\title{
The roasting process and place of cultivation influence the volatile fingerprint of Criollo cocoa from Amazonas, Peru
}

\author{
Marvin G. Valle-Epquín1 ${ }^{\mathbb{D}}$; César R. Balcázar-Zumaeta2 ${ }^{\mathbb{D}}$; Erick A. Auquiñivín-Silva2 ${ }^{\mathbb{D}}$; \\ Armstrong B. Fernández-Jeri2 ${ }^{\mathbb{D}}$; Guillermo Idrogo-Vásquez ${ }^{2}{ }^{\mathbb{D}}$; Efraín M. Castro-Alayo2,*(D) \\ 1 Programa Académico de Ingeniería Agroindustrial, Facultad de Ingeniería y Ciencias Agrarias, Universidad Nacional \\ Toribio Rodríguez de Mendoza de Amazonas, Calle Higos Urco 342-350-356, Chachapoyas, Amazonas. Peru. \\ 2 Instituto de Investigación, Innovación y Desarrollo para el Sector Agrario y Agroindustrial de la Región Amazonas \\ (IIDAA-Amazonas), Facultad de Ingeniería y Ciencias Agrarias, Universidad Nacional Toribio Rodríguez de Mendoza \\ de Amazonas, Calle Higos Urco 342-350-356, Chachapoyas, Amazonas. Peru.
}

Received June 23, 2020. Accepted November 17, 2020.

\begin{abstract}
The Criollo cocoa bean is classified as "fine" or flavor cocoas, being perceived as aromatic or smooth with fruity, raisin, floral, spicy, nutty, molasses, and caramel notes. In the present work, gas chromatography coupled to mass spectrometry was used to study the volatile fingerprint of roasted and unroasted Criollo cocoa from four Amazon districts: Nieva, Cajaruro, Copallín and La Peca located in the Amazon Region of Peru. The results showed that the main sensory perception of cocoa is fruit, and this decreases as the roasting intensity increases. A total of 96 volatile compounds were found, of which the esters had a greater presence in the volatile fingerprint of Criollo cocoa. Propyl acetate (3.5\%), acetoin acetate $(1.3 \%)$ and diethyl succinate $(0.8 \%)$ were found as the characteristic compounds of Criollo cocoa analyzed, which give it its fruit perception. The linalool/benzaldehyde ratio was between 0.56 and 0.89 for La Peca and Cajaruro cocoa. Principal component analysis revealed that the Criollo cocoa in each district has a different volatile fingerprint, whether it is roasted or unroasted beans. The roasting process generates a greater differentiation of the volatile fingerprint of Criollo cocoa.
\end{abstract}

Keywords: Criollo cocoa; roasting; volatile fingerprint; aromatic markers; principal component analysis.

\section{Introduction}

The two main botanical groups of cocoa are the Criollo and the Forastero (Caligiani et al., 2014). The Criollo is classified as "fine" or flavor cocoas, being perceived as aromatic or smooth with fruity, raisin, floral, spicy, nutty, molasses, and caramel notes (Aprotosoaie et al., 2016). In Latin American production, Peruvian cocoa is of interest for its quality; however, its production is insufficient for international demand. In Amazonas, Peru, dry fermented Criollo cocoa bean is produced by the Multi-Service Cooperative APROCAM for the Italian market, and this product has received the designation of the origin "Cacao Amazonas Peru" by the Peruvian State (Castro-Alayo et al., 2019). The popularity of cocoa products is the result of its peculiar flavor, mainly originating from its volatile aromatic fraction (Rottiers et al., 2019b; Ascrizzi et al., 2017; Counet et al., 2002). Most major chocolate manufacturers have premium quality chocolate products in their range, which require fine or flavour cocoa from specific origins in their recipes for the distinct taste or colour of their chocolate (ICCO, 2017).

The sensory quality of cocoa (aroma, taste, mouthfeel, and texture) is the key factor in producing premium products that meet consumer preference (Cordero et al., 2019). The complex volatile profile of cocoa beans, responsible for its unique aroma, is derived from a mixture of more than 600 compounds of different chemical classes, namely alcohols, aldehydes, ketones, acids, esters, and pyrazines (Ziegleder, 2009; Frauendorfer and Schieberle, 2006). Work by Ziegleder (1990) suggested that monoterpenes such as linalool are part of the components or

Cite this article:

Valle-Epquín, M.G.; Balcázar-Zumaeta, C.R.; Auquiñivín-Silva, E.A.; Fernández-Jeri, A.B.; Idrogo-Vásquez, G.; CastroAlayo, E.M. 2020. The roasting process and place of cultivation influence the volatile fingerprint of Criollo cacao from Amazonas-Peru. Scientia Agropecuaria 11(4): 599-610. 
molecules responsible for fine flavour in cocoa and concluded that fine flavour cocoas contain higher amounts of linalool than bulk cocoa. The specific cocoa aroma and final composition of cocoa liquors and chocolates are influenced by many factors, like the cocoa genotype, place of origin of the cocoa beans, season of harvesting, the practices of local farmers, and all subsequent processing steps (Tuenter et al., 2020; Hinneh et al., 2019; Rottiers et al., 2019a; Kongor et al., 2016; Caligiani et al., 2014; Afoakwa et al., 2008). "Terroir" is concerned with the relationship between the characteristics of an agricultural product (quality, taste, style) and its geographic origin, which might influence these characteristics. These effects are challenging to study in other crops such as cocoa where "Terroir" effects similar to wines are implied in many origin specific dark chocolates but have never been systematically tested (Sukha et al., 2017).

The attractive aroma of roasted cocoa is the result of a sophisticated technological process applied to the seeds of the cocoa tree. Among the manufacturing steps, both, fermentation and roasting are considered to be the most important with respect to flavor formation (Frauendorfer and Schieberle, 2008). In particular, roasting is an essential step to develop flavour from the precursors formed during fermentation and drying: the compounds formed depend mainly on temperature and duration of the roasting process (Di Carro et al., 2015). Roasting helps in the removal of undesirable volatile compounds, provides desirable aroma and flavor and makes cocoa beans more brittle (Taş and Gökmen, 2016; loannone et al., 2015). Roasting induces value-added chemical and physical alterations, of which unique colour, aroma and texture are most important (Van Durme et al., 2016). During roasting, some compounds increase in concentration, the volatile fraction decreases, and new compounds are formed (Djikeng et al., 2018; García-Alamilla et al., 2017). The normal convection roasting method is commonly used for roasting cocoa beans at different temperature $\left(120-250^{\circ} \mathrm{C}\right)$ and time varying from for 5 to $120 \mathrm{~min}$ (loannone et al., 2015; Zzaman et al., 2014). Further compounds formed during roasting are pyrazines, aldehydes, alcohols, ethers, furans, thiazoles, pyrones, acids, esters, imines, amines, oxazoles, and pyrroles (Frauendorfer and Schieberle, 2008; Braga et al., 2018).

Since these roasting techniques were introduced to the chocolate industry, the roasting time and temperature have been studied. The results have showed data independently or contradictory to the standpoint of chemical characterization, which leads to the need of specific studies for the type of cocoa by region or roasting system (GarcíaAlamilla et al., 2017; De Brito et al., 2000; Jinap et al., 1998). Literature reviews suggest that the impact of roasting on quality indices should be studied by taking into account the general impact of process parameters; in fact, the degree of cocoa roasting is dependent on both roasting time and temperature (loannone et al., 2015). Therefore, the objective of the present study was studying the influence of the roasting process and place of cultivation on the volatile fingerprint of Criollo cacao in the Amazon-Peru Region within the influence of APROCAM.

\section{Materials and methods}

2.1. Materials

Approximately $14 \mathrm{~kg}$ of dried fermented cocoa beans of Criollo variety were provided directly from Multi-Service Cooperative (APROCAM) in the Bagua province, Amazon region, Perú; specifically from the districts Nieva (17M173770, UTM9465500, 220 m.s.n.m), Cajaruro (17M0801549, UTM9364819, 933 m.s.n.m), Copallín (17M0787843, UTM9372723, 964 m.s.n. m and La Peca (17M0786227, UTM9378161, 1014 m.s.n.m). These districts are within the geographical area of cocoa cultivation administered by APROCAM.

\subsection{Roasting Process}

According to Fernández-Romero et al. (2020), samples of cocoa bean from all districts were subjected to different treatments of time and temperature as conditions of the roasting process $(\mathrm{TO}=$ unrorasted, $\mathrm{T} 1=110$ ${ }^{\circ} \mathrm{C} / 20 \mathrm{~min}, \mathrm{~T} 2=110^{\circ} \mathrm{C} / 35 \mathrm{~min}, \mathrm{~T} 3=140^{\circ} \mathrm{C} / 20$ $\left.\min , \mathrm{T} 4=140^{\circ} \mathrm{C} / 35 \mathrm{~min}\right)$. Prior to the roast, the beans were selected according to their size, choosing beans of uniform size. Criollo cocoa samples $(100 \mathrm{~g})$ were roasted in a roaster (IMSA, ERTC-51, Lima, Peru) in the Agroindustry Plant Pilot at Universidad Nacional Toribio Rodríguez de Mendoza de Amazonas (UNTRM), Peru.

2.3. Analysis of volatile compounds by HSGCMS

2.3.1. Extraction with Solid Phase Micro Extraction (SPME)

According to Álvarez et al. (2016) with some modifications, 5.7 grams of roasted nib cocoa were fragmented in a porcelain mortar and deposited into a $22 \mathrm{~mL}$ vial plus $6.6 \mathrm{~mL}$ of ultrapure water. The vials, were hermetically closed with a septum, leaving it until the equilibrium time be reach. For the analysis, SPME fibres, covered with polydimethylsiloxane (PDMS) film (Supelco, Milan, Italy) with an outer diameter of 50/30 $\mu \mathrm{m}$ 
Carbowax/divinylbenzene (CWX) were used. Before collecting a sample, the fibre was conditioned in the chromatograph injector at $270^{\circ} \mathrm{C}$ for 1 hour and then it was exposed to sample/headspace, at $1 \mathrm{~mm}$ of distance from the interface between the fluid and headspace. Headspace equilibration time was at $50^{\circ} \mathrm{C}, 15 \mathrm{~min}$.

\subsubsection{Gas Chromatographic Analytical Conditions}

The gas chromatograph was an Agilent 7890B Serie II equipped with MSD 5977B and a $60 \mathrm{~m} \times 0.32 \mathrm{~mm} \times 1 \mu \mathrm{m}$ (DB - 5MS UI) capillary column was used through all the work. The GC oven temperature was programmed to $180^{\circ} \mathrm{C} / 5 \mathrm{~min} ; 4^{\circ} \mathrm{C} / \mathrm{min}$ to $250^{\circ} \mathrm{C}$. Helium (flow rate $1.1 \mathrm{~mL} / \mathrm{min}$ ) was the carrier gas; and 55 min run time. Temperature of the injector (operated on split 1:1) and of the detector was $250^{\circ} \mathrm{C}$; the SPME fiber desorption time $5 \mathrm{~min}$. The injected sample volume was $1 \mu \mathrm{L}$.

2.3.3. Identification of the volatile fraction According to Utrilla-Vázquez et al. (2020), identification of the extracted analytes was done by comparison of the corresponding mass spectra in the GC-MS chromatograms with the data of National Institute of Standars and Technology-NIST library. All samples were analyzed in duplicate. A maximal acceptable coefficient of variation was $30 \%$ for a given compound. Aroma descriptors were obtained for each compound detected using the online databases of the Flavor and Extract Manufacturers Association (FEMA) of the United States (FEMA, 2018) and the literature.

\subsection{Statistical analysis}

The roasting process was carried out using the Design of Experiment (DOE) tool of the Minitab 17 software, the experimental runs were done in a random way with two replicas (Table 1). The roasting parameters were time and temperature. The negative signs correspond to the minimum values $(t=20$ $\min , \mathrm{T}=110^{\circ} \mathrm{C}$ ) and the positive signs correspond to the maximum values $(t=35 \mathrm{~min}, T$ $=140^{\circ} \mathrm{C}$ ). A DOE was established for each district.

\section{Table 1}

Experimental design for the roasting process obtained by DOE

\begin{tabular}{cc}
\hline Time, $\min$ & Temperature, ${ }^{\circ} \mathrm{C}$ \\
\hline+ & - \\
+ & - \\
- & + \\
+ & - \\
- & + \\
- & - \\
\hline
\end{tabular}

Analysis of variance was performed for each key aroma compound in all the district. This analysis allowed to obtain the significant volatile compounds of the roasted cocoa. The optimization was done for these key compounds, using Minitab's response optimizer, as follows: for the pleasant volatile compounds the time and temperature that maximized their concentration were found; while for the unpleasant volatile compounds the time and temperature that minimized their concentration was found. According to Rodriguez-Campos et al. (2012), the volatile compounds concentrations were subjected to Principal Component Analysis (PCA) using Minitab 17.

\section{Results and discussion}

Criollo cocoa is of high value and is a fine cocoa used to produce high-quality chocolates. The fine aromas include fruit notes (fresh and ripe), floral, herbal, wood, nuts and caramel notes (Castro-Alayo et al., 2019).

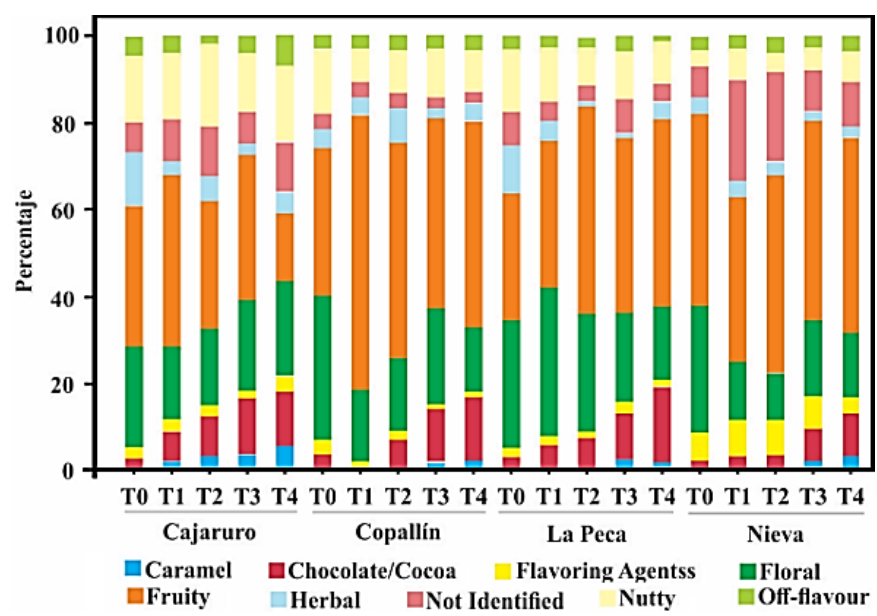

Figure 1. Sensory perception of Criollo cocoa beans during roasting. 
Figure 1 shows the sensory perception of APROCAM Criollo cocoa of all districts according to the intensity of the roasting process. The main perception of the cocoa beans of all the districts is fruit, but this perception decreases as the intensity of roasting increases in Cajaruro cocoa, while the opposite occurs with cocoa from other districts. Tuenter et al. (2020) and Rottiers et al. (2019a) studied the volatile aroma composition of fine-flavor Trinitario, Nacional and Forastero cocoa beans, attribute the fruit characteristic to the presence of 2-heptanol and 2-nonanone. Another perception that the beans of all the districts show is floral, and like the fruit perception.

During fermentation, aroma precursors, such as free amino acids, short-chain peptides, and reducing sugars, are formed, from which the typical cocoa aroma is suggested to be generated during the subsequent roasting process (Frauendorfer and Schieberle, 2008). The main aim of roasting is twofold: the elimination of undesirable compounds with low boiling point, such as acetic acid, and the formation of the typical flavor of roasted beans (Marseglia et al., 2020). Figure 2 shows the chemical classes of the volatile compounds found in APROCAM Criollo cocoa beans. The number of volatile compounds found is a function of the roasting intensity, a greater amount being formed when roasting at a higher intensity, as demonstrated in the Cajaruro T4 treatment (Figure 2a). The high percentage of acidity found in cocoa beans is due to acetic acid, which is found in a higher proportion than the other acids (Figure 2b). The largest number of volatile compounds found in roasted and unroasted beans corresponds to esters, followed by acids, alcohols, aldehydes and ketones. It should be noted that the proportion of esters decreases as the intensity of roasting increases. Therefore, these compounds are characteristic of the cocoa variety, noting that roasting, depending on the time and temperature, reduces their amounts and generates other compounds such as pyrroles, pyrazines and the Strecker aldehydes, coinciding with what was mentioned by Marseglia et al. (2020).

Maillard reactions are of utmost importance during roasting, and initiate reactions between reducing sugars and amino acids. Typical Maillard reaction products include dicarbonyls, heterocyclic compounds, aldehydes formed by Strecker degradation, ketones, esters, alcohols and phenolic compounds. Furthermore, roasting also affects the release of volatile acids (such as acetic acid) (Van Durme et al., 2016). MSfingerprinting on the cocoa headspaces appeared to be a powerful and fast classification technique (Tran et al., 2015). Table 2 shows the volatile compounds identified by HS-SPME-GCMS in APROCAM's Criollo cocoa and its odor description obtained from FEMA. Considering the roasted and unroasted beans, 95 volatile compounds were identified, which were grouped by their chemical class. 40 compounds were identified in the unroasted beans. Aldehydes, 2methylpropanal (burnt, caramel, cocoa, green, malt), 2-isopropyl-5-methyl-2hexenal (floral), 2-phenyl-2-butenal (cocoa, roast, rum), 5-methyl-2-phenyl-2-hexenal (cocoa), were identified in roasted beans, so their presence is due to this process. A high concentration of aldehydes and ketones is desired in cocoa beans (Marseglia et al., 2020), 2-butanone (fragrant, fruit, pleasant), acetoin (butter, creamy, green pepper), 2heptanone (banana-like, fruity) and acetophenone (almonds, flower, meat, must) were produced during roasting. The presence of alcohols increases the quality of the cocoa bean (Rodriguez-Campos et al., 2011), alcohols such as 2-pentanol (fermented, ripe banana), isoamyl alcohol (burnt, cocoa, floral, malt), 2,3-butanediol (sweet, flowery), 2heptanol (citrus, earth, fried, mushroom, oil) and 2-phenethyl alcohol (fruit, honey, lilac, rose, wine) were identified in the beans of all the districts, their presence in beans unroasted means that they were produced in fermentation, most of them produced by yeasts (Marseglia et al., 2020). Other alcohols, such as phenylmethanol (boiled cherries, moss, roasted bread, rose) and 1phenylethanol (floral, honey, rose), were identified in the cocoa beans of Nieva, Cajaruro and La Peca. It is beneficial for aromatic cocoa quality to have 2-phenylethyl acetate and ethylphenyl acetate in high concentrations, due to the flavour notes associated with them (Rodriguez-Campos et al., 2012). 2-phenylethyl acetate (flower, honey, rose) and ethylphenyl acetate (floral, fruit, honey, rose) were found in the beans of all districts. Ethyl 2-methylbutyrate (apple, ester, green apple, kiwi, strawberry) in Copallín and La Peca were identified; hexyl acetate (apple, banana, grass, herb, pear) in Nieva, diethyl succinate (cotton, fabric, floral, fruit, wine) in Nieva and La Peca, ethyl decanoate (brandy, grape, pear) in Nieva (Table 2). The production of these esters can be a result of yeast metabolism during the fermentation process, which produces key cocoa aromas such as flowery and honey flavour notes (Marseglia et al., 2020; 
Aculey et al., 2010; Frauendorfer and Schieberle, 2008).

Pyrazines are considered the most important of the volatile components in cocoa beans. They originate in part by microbiological processes during fermentation but especially by Strecker degradation that accompanies the Maillard reaction during roasting (Marseglia et al., 2020). 2,3dimethylpyrazine (caramel, cocoa, hazelnut, peanut butter, roasted) and 2,3,5trimethylpyrazine (cocoa, earth, must, potato, roast) were the only pyrazines identified in unroasted beans (Table 2), therefore they were produced during fermentation and its concentration increased during roasting. Terpenes are related to the aromatic and antioxidant properties of some plants that contain them (El Atki et al. 2020; Ahmed et al. 2019). Myrcene (balsamic, fruit, geranium, herb, must), 3-carene (lemon), trans-linalool oxide (floral) and linalool (coriander, floral, lavender, lemon, rose) were identified in the beans roasted and unroasted from all districts except Nieva, which was found only in unroasted beans and then disappeared during all treatments (Table 2). The presence of these terpenes could be related to the antioxidant properties of the studied cocoa. These compounds were also identified in Criollo cocoa from other geographic locations studied by Qin et al. (2016).

According to Utrilla-Vázquez et al. (2020), key-aroma markers and technological markers determining the aromatic properties and quality of cocoa beans. The 16 key-aroma markers and technological markers finding by Utrilla-Vázquez et al. (2020) were finding in APROCAM Criollo cocoa beans (Figure 3). Acetic acid (acid, fruit, pungent, sour, vinegar) is considered an unpleasant volatile compounds, was found in values higher than
$30 \%$ in the roasted beans (T2) of Copallín, followed by Nieva with values higher than $25 \%$, while the lowest percentage of acetic acid it was obtained in the roasted beans (T4) of Cajaruro; which indicates that a higher roasting intensity allows the release of acetic acid.

This is in accordance, with Tuenter et al. (2020) who analyzed National cocoa liquor from Ecuador and Forastero form West Africa, finding acetic acid as the dominant compound in both samples. The aldehyde Strecker 3-methylbutanal has been observed a compound quite often proposed as an important aroma compound in roasted cocoa (Frauendorfer and Schieberle, 2008), the highest concentration of 3methylbutanal was found in the Cajaruro cocoa beans, followed by Copallín, La Peca and Nieva. In these districts, the concentration of 3-methylbutanal was increased according to the intensity of the roasting process (Figure 3 ), coinciding with Frauendorfer and Schieberle (2008) who suggest that these odorants should contribute most to the changes in the overall aroma after roasting. Considering that the statistical model of 3-methylbutanal for the districts of Cajaruro, Nieva and La Peca was not significant, it was only possible to optimize the roasting process for Copallín beans, in which roasting at $122.4^{\circ} \mathrm{C}$ for 35 minutes was necessary to maximize the concentration of 3-methylbutanal up to $6.54 \%$ (Table 3). More intense roasting parameters will cause the reduction of the Strecker aldehyde content, since as explained by Afoakwa (2010), the degradation of these aldehydes can serve as precursors of other types of pyrazines due to heterocyclization reactions, being verified by Hinneh et al. (2019).

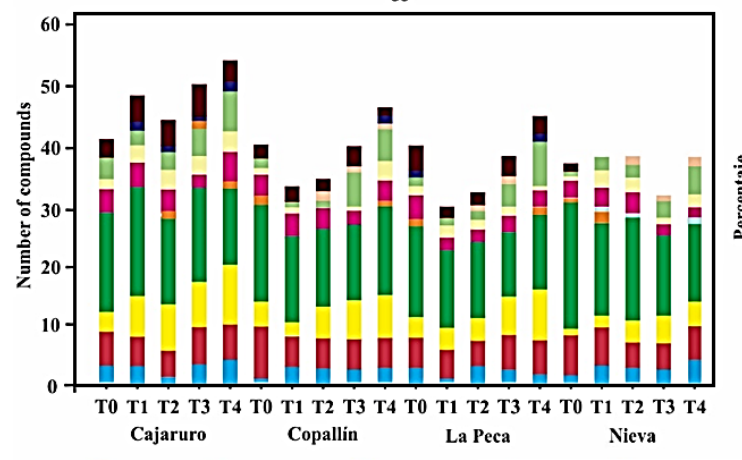

b

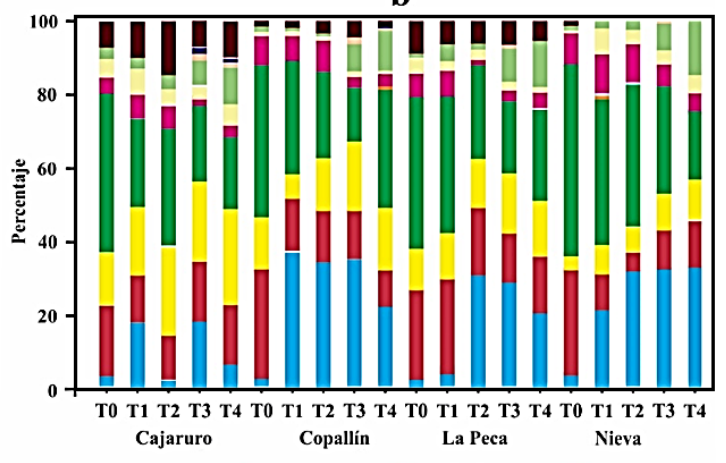

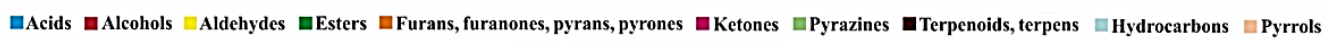
a Sulfur compounds Others

Figure 2. Volatile compounds identified by HS-SPME GC-MS during the roasting process of Criollo cocoa beans. Number of identified compounds (a) and concentration of chemical classes (b). 
Table 2

Volatile compounds of Criollo cocoa beans during roasting process

\begin{tabular}{|c|c|c|c|c|c|c|c|c|c|c|c|c|c|c|c|c|c|c|c|c|c|c|}
\hline \multirow{2}{*}{ Code } & \multirow{2}{*}{ Compound } & \multirow{2}{*}{ Description } & \multicolumn{4}{|c|}{ Nieva } & \multicolumn{6}{|c|}{ Cajaruro } & \multicolumn{5}{|c|}{ Copallín } & \multicolumn{5}{|c|}{ La Peca } \\
\hline & & & T0 & T1 & T2 & T3 & T4 & T0 & T1 & T2 & T3 & T4 & TO & T1 & T2 & T3 & T4 & T0 & T1 & & T3 & $\mathrm{T} 4$ \\
\hline & $\begin{array}{l}\text { Aldehydes } \\
\text { 2-methylpropanal }\end{array}$ & & & & & & & & & & & & & & & & & & & & & \\
\hline $\begin{array}{l}\text { A1 } \\
\text { A2 }\end{array}$ & $\begin{array}{l}\text { 2-methylpropanal } \\
\text { 3-methylbutanal }\end{array}$ & Burnt, caramel, cocoa, green, malt [a] & & & & & & & $\begin{array}{l}x \\
x\end{array}$ & 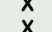 & 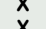 & $\begin{array}{l}x \\
x\end{array}$ & & & & & & & & & & \\
\hline A3 & $\begin{array}{l}\text { 3-methylbutanal } \\
\text { 2-methylbutyraldehyde }\end{array}$ & $\begin{array}{l}\text { Chocolate [a] } \\
\text { Almond, cocoa, fermented, hazelnut, malt [a] }\end{array}$ & & $x$ & $\begin{array}{l}x \\
x\end{array}$ & $\begin{array}{c}x \\
x\end{array}$ & $\begin{array}{l}x \\
x\end{array}$ & $\begin{array}{l}x \\
x\end{array}$ & $\begin{array}{l}x \\
x\end{array}$ & $\begin{array}{c}x \\
x\end{array}$ & $\begin{array}{l}x \\
x\end{array}$ & $\begin{array}{c}x \\
x\end{array}$ & $\begin{array}{l}x \\
x\end{array}$ & $x$ & $\begin{array}{l}x \\
x\end{array}$ & $\begin{array}{l}x \\
x\end{array}$ & $\begin{array}{l}x \\
x\end{array}$ & $\begin{array}{l}x \\
x\end{array}$ & $\begin{array}{l}x \\
x\end{array}$ & $x_{y}$ & $\underset{x}{x}$ & 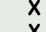 \\
\hline A4 & 3-furancarboxaldehyde & & & $x$ & & & & & & & & $\hat{x}$ & & & $\hat{x}$ & & & & & & & $\begin{array}{l}x \\
x\end{array}$ \\
\hline A5 & 5-methylfurfural & Flavoring agents $[\mathrm{a}]$ & & & & & & & & & & $\hat{x}$ & & & & & $\mathrm{x}$ & & & & & $\hat{x}$ \\
\hline A6 & Benzaldehyde & Sweet, bitter almond, cherry [a] & $\mathrm{x}$ & $\mathrm{x}$ & $\mathrm{x}$ & $\mathrm{x}$ & $\mathrm{x}$ & $\mathrm{x}$ & $\mathrm{x}$ & $\mathrm{x}$ & $\mathrm{x}$ & $\hat{x}$ & $\mathrm{x}$ & $\mathrm{x}$ & $\mathrm{x}$ & $\mathrm{x}$ & $\hat{x}$ & $\mathrm{x}$ & $\mathrm{x}$ & $\mathrm{x}$ & $x$ & $\hat{x}$ \\
\hline A7 & Benzeneacetaldehyde & Berry, geranium, honey, nut, pungent [a] & $\hat{x}$ & $\hat{x}$ & $\hat{x}$ & $\hat{x}$ & $\hat{x}$ & $\hat{x}$ & $\hat{x}$ & $\hat{x}$ & $\hat{x}$ & $\hat{x}$ & $\hat{x}$ & $\hat{x}$ & $\hat{x}$ & $\hat{x}$ & $\hat{x}$ & $\hat{x}$ & $\hat{x}$ & $\hat{x}$ & $\hat{x}$ & $\hat{x}$ \\
\hline A8 & 2-isopropyl-5-methyl-2-hexenal & Floral [a] & & & & $x$ & $x$ & & $x$ & $x$ & $x$ & $x$ & & & & $x$ & $x$ & & & & $x$ & $x$ \\
\hline A9 & 2-phenyl-but-2-enal & Cocoa, roast, rum [a] & & & & & & & $\hat{x}$ & $\hat{x}$ & $\hat{x}$ & $\hat{x}$ & & & & $\hat{x}$ & $\hat{x}$ & & & & $\hat{x}$ & $\ddot{x}$ \\
\hline A10 & 5-methyl-2-phenyl-2-hexenal & Cocoa [a] & & & & & & & & & $x$ & $x$ & & & & & & & & & & \\
\hline & Ketones & & & & & & & & & & & & & & & & & & & & & \\
\hline K1 & Diacetyl & Butter, pastry, yeast [a] & & & & & & & & & & $\mathrm{x}$ & & & & & & & & & & \\
\hline K2 & 2-butanone & Fragrant, fruit, pleasant [a] & & & & & & & & & & $\hat{x}$ & & & & & & & & & & \\
\hline K3 & 2-pentanone & Fruit, pungent [a] & & & & & & & & & & $\hat{x}$ & & & & & & & & & & \\
\hline $\mathrm{K} 4$ & 3-methyl-2-butanone & Flavoring agents [a] & & & & & & $\mathrm{x}$ & $\mathrm{x}$ & $x$ & & & $\mathrm{x}$ & & & & & $x$ & & & & $x$ \\
\hline K5 & Acetoin & Butter, creamy, green pepper [a] & $\mathrm{x}$ & $\mathrm{x}$ & $\mathrm{x}$ & $\mathrm{x}$ & $x$ & $\ddot{x}$ & & $\hat{x}$ & & & & $\mathrm{x}$ & $\mathrm{x}$ & & $\mathrm{x}$ & & & & $\mathrm{x}$ & \\
\hline K6 & 2-heptanone & Banana-like, fruity [b] & $\mathrm{x}$ & $\mathrm{x}$ & $\ddot{x}$ & $\mathrm{x}$ & $\mathrm{x}$ & $\mathrm{x}$ & $\mathrm{x}$ & $\mathrm{x}$ & $\mathrm{x}$ & $\mathrm{x}$ & $\mathrm{x}$ & $\mathrm{x}$ & $\mathrm{x}$ & $\mathrm{x}$ & $\ddot{x}$ & $\mathrm{x}$ & $\mathrm{x}$ & $\mathrm{x}$ & & $\mathrm{x}$ \\
\hline K7 & Acetophenone & Almonds, flower, meat, must [a] & & & & & & $\hat{x}$ & $\hat{x}$ & $\hat{x}$ & $\hat{x}$ & $\hat{x}$ & $\hat{x}$ & $\hat{x}$ & $\hat{x}$ & $\hat{x}$ & $\hat{x}$ & $\hat{x}$ & $\hat{x}$ & $\hat{x}$ & $\mathrm{x}$ & $\hat{x}$ \\
\hline K8 & 3-(hydroxymethyl)-2-nonanone & & $x$ & $\mathrm{x}$ & $\mathrm{x}$ & & & & $x$ & & & & $x$ & $\mathrm{x}$ & $\mathrm{x}$ & & & $\mathrm{x}$ & & & & \\
\hline & Alcohols & & & & & & & & & & & & & & & & & & & & & \\
\hline AL1 & 3-methyl-2-butanol & Flavoring agents [a] & & & $x$ & & & & & & & $x$ & & & & & $x$ & & & & $x$ & \\
\hline AL2 & 2-pentanol & Fermented, ripe banana [a] & $\mathrm{x}$ & $\mathrm{x}$ & & & $x$ & $x$ & $\mathrm{x}$ & $\mathrm{x}$ & $\mathrm{x}$ & & $\mathrm{x}$ & $\mathrm{x}$ & $\mathrm{x}$ & & & $x$ & $\mathrm{x}$ & & & $\mathrm{x}$ \\
\hline AL3 & Isoamyl alcohol & Burnt, cocoa, floral, malt [a] & $\mathrm{x}$ & $\mathrm{x}$ & & & $\mathrm{x}$ & $\mathrm{x}$ & $\mathrm{x}$ & & $\mathrm{x}$ & $\mathrm{x}$ & $\mathrm{x}$ & $\mathrm{x}$ & $x$ & $\mathrm{x}$ & $\mathrm{x}$ & $\mathrm{x}$ & & $\mathrm{x}$ & & $\mathrm{x}$ \\
\hline AL4 & Amyl alcohol & Balsamic, fruit, green, pungent, yeast [a] & & $\hat{x}$ & $\mathrm{x}$ & $\mathrm{x}$ & & & & $\mathrm{x}$ & & & $\hat{x}$ & & & $\hat{x}$ & & & $\mathrm{x}$ & $\hat{x}$ & $\mathrm{x}$ & $\hat{x}$ \\
\hline AL5 & 2,3-butanediol & Sweet, flowery [b] & $\mathrm{x}$ & $\hat{x}$ & $\ddot{x}$ & $\ddot{x}$ & $\mathrm{x}$ & $\mathrm{x}$ & $\mathrm{x}$ & $\hat{x}$ & $\mathrm{x}$ & $\mathrm{x}$ & $\hat{x}$ & $\mathrm{x}$ & $\mathrm{x}$ & $\hat{x}$ & $\mathrm{x}$ & $\mathrm{x}$ & $\hat{x}$ & $\hat{x}$ & $\ddot{x}$ & $\ddot{x}$ \\
\hline AL6 & 2-heptanol & Citrus, earth, fried, mushroom, oil [a] & $\hat{x}$ & & & & $\hat{x}$ & $\hat{x}$ & $\hat{x}$ & $\hat{x}$ & $\hat{x}$ & $\hat{x}$ & $\hat{x}$ & $\hat{x}$ & $\hat{x}$ & $\hat{x}$ & $\hat{x}$ & $\hat{x}$ & $\hat{x}$ & $\hat{x}$ & $\hat{x}$ & $\hat{x}$ \\
\hline AL7 & 2-butoxyethanol & Flavoring agents [a] & & & & & & & & & & $\hat{x}$ & $\hat{x}$ & & & & & & & & & \\
\hline AL8 & Phenylmethanol & Boiled cherries, moss, roasted bread, rose [a] & & $\mathrm{x}$ & & & & & & & & & & & & & & & & & & \\
\hline AL9 & 1-phenylethanol & Floral, honey, rose [a] & & & & & & $\mathrm{x}$ & & $x$ & $\mathrm{x}$ & & $\mathrm{x}$ & & & & & & & & & \\
\hline AL10 & 2-nonanol & Cucumber [a] & $x$ & $\mathrm{x}$ & $\mathrm{x}$ & $\mathrm{x}$ & $\mathrm{x}$ & & $x$ & & & & $\mathrm{x}$ & & & & & & & & & \\
\hline AL11 & 2-phenethyl alcohol & Fruit, honey, lilac, rose, wine [a] & $\hat{x}$ & & & $\hat{x}$ & $\hat{x}$ & $\mathrm{x}$ & & & $x$ & $\mathrm{x}$ & $\hat{x}$ & $\mathrm{x}$ & $\mathrm{x}$ & $x$ & $x$ & $\mathrm{x}$ & $x$ & $x$ & $\mathrm{x}$ & $\mathrm{x}$ \\
\hline & Esters & & & & & & & & & & & & & & & & & & & & & \\
\hline E1 & Methyl acetate & Ester, green [a] & & $x$ & & & $\mathrm{x}$ & $x$ & & $x$ & $\mathrm{x}$ & $x$ & $\mathrm{x}$ & & & $\mathrm{x}$ & & & $x$ & & & $x$ \\
\hline E2 & Ethyl ethanoate & Pineapple [c] & $x$ & & & & & $\ddot{x}$ & & & & & $x$ & $\mathrm{x}$ & & & $x$ & & & & & \\
\hline E3 & Vinyl acetate & Flavoring agents [a] & & & & & & & & & $x$ & & & & & & & & & & & \\
\hline E4 & Propyl acetate & Celery, floral, pear, red fruit [a] & $\mathrm{x}$ & $\mathrm{x}$ & $\mathrm{x}$ & $x$ & $x$ & & $\mathrm{x}$ & $x$ & $x$ & & & & & & & & $x$ & $x$ & $x$ & \\
\hline E5 & 2-butyl acetate & Flavoring agents [a] & $x$ & $x$ & $\mathrm{x}$ & $\ddot{x}$ & $\ddot{x}$ & & & & & & & & & & & & & & & \\
\hline E6 & Isobutyl acetate & Apple, aanana, floral, herb [a] & $x$ & $x$ & $x$ & $x$ & $x$ & $x$ & $x$ & $x$ & $\mathrm{x}$ & $\mathrm{x}$ & $\mathrm{x}$ & $\mathrm{x}$ & $\mathrm{x}$ & $x$ & $x$ & $x$ & $x$ & $\mathrm{x}$ & $x$ & $x$ \\
\hline E7 & Butyl acetate & Apple, aanana, glue, pungent [a] & & & & & & & $\ddot{x}$ & & & & & & & & & & & & & \\
\hline E8 & 1-methylbutyl acetate & Fruit [a] & $\mathrm{x}$ & $\mathrm{x}$ & $\mathrm{x}$ & $\mathrm{x}$ & $\mathrm{x}$ & $\mathrm{x}$ & $\mathrm{x}$ & $\mathrm{x}$ & $\mathrm{x}$ & $\mathrm{x}$ & $\mathrm{x}$ & $\mathrm{x}$ & $\mathrm{x}$ & $\mathrm{x}$ & $x$ & $x$ & $x$ & $\mathrm{x}$ & $\mathrm{x}$ & $x$ \\
\hline E9 & Ethyl 2-methylbutyrate & Apple, ester, green apple, kiwi, strawberry [a] & & & & & & & & & & & $\mathrm{x}$ & & & & & & & & & \\
\hline E10 & Ethyl isovalerate & Apple, fruit, Pineapple, Sour [a] & & $\mathrm{x}$ & $\mathrm{x}$ & & & $\mathrm{x}$ & $\mathrm{x}$ & $x$ & $\mathrm{x}$ & & $\ddot{x}$ & & & $\mathrm{x}$ & $\mathrm{x}$ & $\mathrm{x}$ & & & & \\
\hline E11 & 3-methylbutyl acetate & Apple, banana, glue, pear [a] & & & & & & $\hat{x}$ & & & & & & $x$ & & & $\mathrm{x}$ & & & & & $\mathrm{x}$ \\
\hline E12 & 2-methylbutyl acetate & Apple, banana, pear [a] & $x$ & & & & & $\ddot{x}$ & $x$ & $x$ & $\mathrm{x}$ & $x$ & $x$ & $\ddot{x}$ & $x$ & $x$ & $\ddot{x}$ & $x$ & $x$ & $x$ & $x$ & $x$ \\
\hline E13 & Amyl acrylate & & & $\mathrm{x}$ & $x$ & & & & & & & & & & & & & & & & & \\
\hline E14 & Acetoin acetate & Fruit [a] & $\mathrm{x}$ & $\hat{x}$ & $\hat{x}$ & $\mathrm{x}$ & $\mathrm{x}$ & & $\mathrm{x}$ & $\mathrm{x}$ & $\mathrm{x}$ & $\mathrm{x}$ & $\mathrm{x}$ & $\mathrm{x}$ & $\mathrm{x}$ & $x$ & $\mathrm{x}$ & $\mathrm{x}$ & $\mathrm{x}$ & $\mathrm{x}$ & $\mathrm{x}$ & $\mathrm{x}$ \\
\hline E15 & Pentyl acetate & Flavoring agents [a] & & & & & & & $x$ & & & & & & & & & & & & & \\
\hline E16 & Prenyl acetate & Putty [a] & & & & & & & $\hat{x}$ & & & & & & & & & $x$ & & & & \\
\hline E17 & 3-ethoxypropyl acetate & & & & & & & & $\ddot{x}$ & $\mathrm{x}$ & $\mathrm{x}$ & & & $x$ & $\mathrm{x}$ & & & & & & & \\
\hline E18 & Ethyl hexanoate & Apple peel, brandy, fruit gum, overripe fruit, pineapple [a] & $\mathrm{x}$ & $\mathrm{x}$ & $\mathrm{x}$ & $x$ & $\mathrm{x}$ & $\mathrm{x}$ & $\hat{x}$ & $\hat{x}$ & $\hat{x}$ & $\mathrm{x}$ & $\mathrm{x}$ & $\hat{x}$ & $\hat{x}$ & $x$ & $\mathrm{x}$ & $\mathrm{x}$ & $\mathrm{x}$ & $\mathrm{x}$ & $\mathrm{x}$ & $\mathbf{x}$ \\
\hline E19 & Hexyl acetate & Apple, banana, grass, herb, pear [a] & $\mathrm{x}$ & & $\mathrm{x}$ & & & & & & & & & & & & & & & & & \\
\hline
\end{tabular}




\begin{tabular}{|c|c|c|c|c|c|c|c|c|c|c|c|c|c|c|c|c|c|c|c|c|c|c|}
\hline E20 & 2-heptyl acetate & Flavoring agents [a] & $\mathrm{x}$ & $\mathrm{x}$ & $\mathrm{x}$ & $x$ & $x$ & $x$ & $x$ & $\mathrm{x}$ & $\mathrm{x}$ & $x$ & $x$ & $x$ & $\mathrm{x}$ & $x$ & $x$ & $x$ & $x$ & $x$ & $x$ & $\mathrm{x}$ \\
\hline E21 & 2,3-butanediol, diacetate & & $x$ & $\mathrm{x}$ & $x$ & $\mathrm{x}$ & $\mathrm{x}$ & $x$ & $x$ & $\mathrm{x}$ & $\mathrm{x}$ & $\mathrm{x}$ & $\mathrm{x}$ & $\mathrm{x}$ & $\mathrm{x}$ & $\mathrm{x}$ & $\mathrm{x}$ & $x$ & $\mathrm{x}$ & $x$ & $x$ & $\mathrm{x}$ \\
\hline E22 & Methoxyacetic acid, 3-methylbutyl ester & & & & & & & & & $\hat{x}$ & & & & & & & & & & & & \\
\hline E23 & 2-butoxyethyl acetate & & & & & & & & $x$ & & & & $\mathrm{x}$ & & & & & $\mathrm{x}$ & & & & \\
\hline E24 & Trimethylene acetate & & $x$ & & $x$ & & & $x$ & $\mathrm{x}$ & $x$ & $x$ & $\mathrm{x}$ & & & $\mathrm{x}$ & $\mathrm{x}$ & $\mathrm{x}$ & & & & & \\
\hline E25 & Linalyl formate & Citrus, coriander [a] & $x$ & & & & & $\mathrm{x}$ & & & & & $\mathrm{x}$ & $\mathrm{x}$ & $\mathrm{x}$ & & $x$ & $x$ & & & & \\
\hline E26 & Benzyl acetate & Fruit [a] & & & $x$ & $x$ & & & & & & $\mathrm{x}$ & & & & & & & & & & \\
\hline E27 & Diethyl succinate & Cotton, fabric, floral, fruit, wine [a] & $x$ & $\mathrm{x}$ & $x$ & $\mathrm{x}$ & $\mathrm{x}$ & & & & & & & & & & & & $\mathrm{x}$ & $\mathrm{x}$ & $x$ & $x$ \\
\hline E28 & Ethyl benzoate & Camomile, celery, fat, flower, fruit [a] & $\mathrm{x}$ & $\mathrm{x}$ & $x$ & $x$ & $\mathrm{x}$ & $x$ & $x$ & & $x$ & $\mathrm{x}$ & & & & & & $x$ & & & & \\
\hline E29 & Ethyl octanoate & Apricot, brandy, fat, floral, pineapple [a] & $\mathrm{x}$ & $\mathrm{x}$ & $\mathrm{x}$ & $\mathrm{x}$ & $\mathrm{x}$ & $\mathrm{x}$ & $\mathrm{x}$ & $\mathrm{x}$ & $\mathrm{x}$ & $\mathrm{x}$ & $\mathrm{x}$ & $\mathrm{x}$ & $\mathrm{x}$ & $\mathrm{x}$ & $x$ & $\mathrm{x}$ & $x$ & $\mathrm{x}$ & $\mathrm{x}$ & $\mathrm{x}$ \\
\hline E30 & Ethylphenyl acetate & Floral, fruit, honey, rose [a] & $\hat{x}$ & $\hat{x}$ & $\hat{x}$ & $\hat{x}$ & $\hat{x}$ & $\hat{x}$ & $\hat{x}$ & $\hat{x}$ & $\hat{x}$ & $\hat{x}$ & $\hat{x}$ & $\hat{x}$ & $\hat{x}$ & $\hat{x}$ & $\hat{x}$ & $\hat{x}$ & $\hat{x}$ & $\hat{x}$ & $\hat{x}$ & $\hat{x}$ \\
\hline E31 & 2-phenylethyl acetate & Flower, honey, rose [a] & $\hat{x}$ & & & & & $\hat{x}$ & & & & & $\hat{x}$ & $\hat{x}$ & $\hat{x}$ & $\hat{x}$ & $\hat{x}$ & $\hat{x}$ & $\hat{x}$ & $\hat{x}$ & $\hat{x}$ & $\hat{x}$ \\
\hline \multirow{3}{*}{$\begin{array}{l}\text { E32 } \\
\text { E33 }\end{array}$} & Ethyl decanoate & Brandy, grape, pear [a] & $x$ & & & & & & & & & & & & & & & & & & & \\
\hline & 1-methylbutyl benzoate & & $\mathrm{x}$ & & & & & & & & & & & & & & & & & & & \\
\hline & $\begin{array}{l}\text { Acids } \\
\text { Acetic acid }\end{array}$ & Acid, fruit, pungent, sour, vinegar [a] & & $x$ & $x$ & $\mathrm{x}$ & $x$ & & $x$ & & $x$ & & & $\mathrm{x}$ & $\mathrm{x}$ & $x$ & $x$ & & & $\mathrm{x}$ & $x$ & $\mathrm{x}$ \\
\hline AC2 & Isobutyric acid & Burnt, butter, cheese, sweat, rancid [a] & & $n$ & $\mathrm{n}$ & $\hat{n}$ & $\hat{x}$ & $x$ & $\mathrm{n}$ & & n & $\mathrm{x}$ & & $n$ & $\hat{n}$ & $\hat{n}$ & 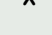 & & & n & $\hat{n}$ & n \\
\hline AC3 & Isovaleric acid & Cheese, pungent, sweat, rancid [a] & $\mathrm{x}$ & $\mathrm{x}$ & $\mathrm{x}$ & $\mathrm{x}$ & $\ddot{x}$ & $\mathrm{x}$ & $x$ & & $x$ & $\hat{x}$ & $x$ & $\mathrm{x}$ & $x$ & $x$ & $x$ & $\mathrm{x}$ & $\mathrm{x}$ & $\mathrm{x}$ & $x$ & $\mathrm{x}$ \\
\hline \multirow{3}{*}{$\begin{array}{l}\text { AC4 } \\
\text { AC5 }\end{array}$} & 2-methylbutanoic acid & Butter, cheese, fermented, sour [a] & $\mathrm{x}$ & $\mathrm{x}$ & $\mathrm{x}$ & $\mathrm{x}$ & $\mathrm{x}$ & $\mathrm{x}$ & $\mathrm{x}$ & $x$ & $\mathrm{x}$ & $\mathrm{x}$ & & $\mathrm{x}$ & $\mathrm{x}$ & $\mathrm{x}$ & $x$ & $\mathrm{x}$ & & $\mathrm{x}$ & $x$ & \\
\hline & 4-hydroxybutanoic acid & & & & & & & & & & & $\hat{x}$ & & & & & & $\hat{x}$ & & & & \\
\hline & Pyrazines & & & & & & & & & & & & & & & & & & & & & \\
\hline P1 & Methylpirazine & Cocoa, green, hazelnut, popcorn, roasted [a] & & & & & & & & & & & & & & & & & & & & $x$ \\
\hline P2 & 2,5-dimethylpyrazine & Cocoa, roast beef, roasted nut [a] & & & & & $\mathrm{x}$ & & & & $\mathrm{x}$ & $\mathrm{x}$ & & & & $\mathrm{x}$ & $\mathrm{x}$ & & & & $x$ & $x$ \\
\hline P3 & 2-ethylpyrazine & Burnt, green, iron scorch, must, peanut butter, roasted, rum, wood [ & & & & & & & & & & $\mathrm{x}$ & & & & & & & & & & \\
\hline P4 & 2,3-dimethylpyrazine & Caramel, cocoa, hazelnut, peanut butter, roasted [a] & & & & & $\mathrm{x}$ & $\mathrm{x}$ & $x$ & $x$ & & $\ddot{x}$ & $\mathrm{x}$ & & & $\mathrm{x}$ & $x$ & & & & & $\mathrm{x}$ \\
\hline P5 & 2-ethyl-6-methylpyrazine & Cocoa, roasted, green [d] & & & & & & & & & $\mathrm{x}$ & $x$ & & & & $x$ & $x$ & & & & & $x$ \\
\hline P6 & $2,3,5$-trimethylpyrazine & Cocoa, earth, must, potato, roast [a] & $\mathrm{x}$ & $\mathrm{x}$ & $\mathrm{x}$ & $\mathrm{x}$ & $\mathrm{x}$ & $\mathrm{x}$ & $\mathrm{x}$ & $\mathrm{x}$ & 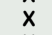 & & $\mathrm{x}$ & $\mathrm{x}$ & $\mathrm{x}$ & $x$ & $\ddot{x}$ & $\mathrm{x}$ & $\mathrm{x}$ & $\mathrm{x}$ & $\mathrm{x}$ & $x$ \\
\hline P7 & 3-ethyl-2,5-dimethylpyrazine & Earthy, roasted [d] & & & & $x$ & $x$ & & & & $x$ & $x$ & & & & $x$ & $x$ & & & & $x$ & $x$ \\
\hline \multirow[t]{3}{*}{ P8 } & Ethyltrimethylpyrazine & Candy, sweet [c] & & $\mathrm{x}$ & $\mathrm{x}$ & $x$ & $\mathrm{x}$ & $\mathrm{x}$ & $\mathrm{x}$ & $\mathrm{x}$ & $\mathrm{x}$ & $\mathrm{x}$ & & & & $x$ & $x$ & & & & $\mathrm{x}$ & $\mathrm{x}$ \\
\hline & Terpenoids y terpenes & & & & & & & & & & & & & & & $x$ & $\ddot{x}$ & & & & & \\
\hline & Myrcene & Balsamic, fruit, geranium, herb, must [a] & $\mathrm{x}$ & & & & & $\mathrm{x}$ & $\mathrm{x}$ & $\mathrm{x}$ & $\mathrm{x}$ & $\mathrm{x}$ & $\mathrm{x}$ & $\mathrm{x}$ & $x$ & $\hat{x}$ & $\hat{x}$ & $x$ & $x$ & $\mathrm{x}$ & & $\mathrm{x}$ \\
\hline T2 & 3-carene & Lemon [a] & $n$ & & & & & $\hat{x}$ & $\hat{x}$ & $\hat{x}$ & $\hat{x}$ & $n$ & $n$ & $n$ & $\hat{x}$ & 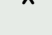 & $\mathrm{n}$ & $\hat{x}$ & $n$ & $n$ & & n \\
\hline T3 & Trans-linalool oxide & Floral [e] & & & & & & & & & $x$ & $\mathrm{x}$ & & $\mathrm{x}$ & & $\mathrm{x}$ & & $\mathrm{x}$ & & & $x$ & \\
\hline T4 & Linalool & Coriander, floral, lavender, lemon, rose [a] & & & & & & & $\mathrm{x}$ & $x$ & $\mathrm{x}$ & $\mathrm{x}$ & & & & $\mathrm{x}$ & & & $\mathrm{x}$ & $\mathrm{x}$ & $x$ & $\mathrm{x}$ \\
\hline T5 & Alloocimene & Flavoring agents [a] & & & & & & $\mathrm{x}$ & $x$ & & $x$ & $\mathrm{x}$ & & & & & & $x$ & & & $\mathrm{x}$ & $x$ \\
\hline T6 & $\begin{array}{l}\text { Alpha-pyronene } \\
\text { Pyrrols }\end{array}$ & & & & & & & & & $\mathrm{x}$ & & & & & & & & & & & & \\
\hline P1 & 2-Acetylpyrrole & Bread, Cocoa, Hazelnut, Licorice, Walnut [a] & & & $\mathrm{x}$ & $\mathrm{x}$ & $\mathrm{x}$ & & & & $\mathrm{x}$ & $\mathrm{x}$ & & & $\mathrm{x}$ & $x$ & $\mathrm{x}$ & & & $\mathrm{x}$ & $\mathrm{x}$ & \\
\hline & Furans, furanones, pyrans, pyrones & & & & & & & & & & & & & & & & & & & & & \\
\hline \multirow{4}{*}{$\begin{array}{l}\text { F1 } \\
\text { F2 } \\
\text { F3 }\end{array}$} & 2-Ethyl-5-methylfuran & Flavoring Agents [a] & $\mathrm{x}$ & $\mathrm{x}$ & & & & & & & & & $\mathrm{x}$ & & & & & $x$ & & & & \\
\hline & 2-Acetylfuran & Balsamic, Cocoa, Coffee [a] & & & & & & & & & & $\mathrm{x}$ & & & & & $\mathrm{x}$ & & & & & $\mathrm{x}$ \\
\hline & $\begin{array}{l}\text { Butyrolactone } \\
\text {. }\end{array}$ & Caramel, Cheese, Roasted Nut [a] & & $\mathrm{x}$ & & & & & & $\mathrm{x}$ & & & & & & & & & & & & \\
\hline & Sulfure compounds & & & & & & & & & & & & & & & & & & & & & \\
\hline \multirow{2}{*}{$\begin{array}{l}\text { S1 } \\
\text { S2 } \\
\text { s3 }\end{array}$} & $\begin{array}{l}\text { Dimethyl sulfide } \\
\text { Dimethyl sulfone }\end{array}$ & Cabbage, Organic, Sulfur, Wet Earth [a] & & & & & & & $x$ & $x$ & $x$ & $x$ & & & & & & $x$ & & & & $\mathrm{x}$ \\
\hline & Disulfide, dimethyl & Cabbage, Garlic, Onion [a] & & & & & & & & & & & & & & & $\mathrm{x}$ & & & & & \\
\hline \multirow[t]{2}{*}{ H1 } & $\begin{array}{l}\text { Hydrocarbons } \\
\text { Styrene }\end{array}$ & Flavoring Agents [a] & & $\mathrm{x}$ & $\mathrm{x}$ & & $\mathrm{x}$ & & & & & & & & & & & & & & & \\
\hline & Others & & $x$ & $x$ & $x$ & & & $x$ & $x$ & $x$ & $x$ & $x$ & $x$ & & & & & $x$ & & & & \\
\hline 02 & $\begin{array}{l}\text { 1,3-Dioxolane, , 2,4,5-trimetnyl- } \\
\text { Dimethoxymethane }\end{array}$ & Flavoring Agents [a] & $\hat{n}$ & A & $\boldsymbol{n}$ & & & $\hat{n}$ & $\hat{n}$ & A & $\hat{n}$ & $\hat{n}$ & $\hat{n}$ & & & & & $\hat{n}$ & $\mathrm{x}$ & $\mathrm{x}$ & & \\
\hline 03 & Heptyl tiglate, $4-$ & & & $\mathrm{x}$ & $\mathrm{x}$ & $\mathrm{x}$ & $\mathrm{x}$ & & & & & & & & & & & & & & & \\
\hline 04 & 1H-1,2,4-Triazol-5-amine, 1-methyl- & & & & & & $\mathrm{x}$ & & & & $\mathrm{x}$ & $\mathrm{x}$ & & & & & $\mathrm{x}$ & & & & $\mathrm{x}$ & \\
\hline O5 & Bicyclo[3.1.1]hept-2-ene, 3,6,6-trimethyl- & Unronan-2-y carbonate & & $x$ & & & & $x$ & $\begin{array}{l}x \\
x \\
x\end{array}$ & $\begin{array}{l}x \\
x\end{array}$ & $x$ & $x$ & $x$ & $x$ & & $x$ & $\begin{array}{l}x \\
x\end{array}$ & $x$ & $x$ & $x$ & $x$ & $x$ \\
\hline 06 & Ethyl 2-(5-methyl-5-vinyltetrahydrofuran-2-1 & 1) propan-2-yl carbonate & & $x$ & $x$ & & & & $x$ & $x$ & & & & & & & $x$ & & & & & \\
\hline
\end{tabular}


Isovaleric acid (cheese, pungent, sweat, rancid) is considered an unpleasant volatile compounds, may be formed by aerobic putrefactive bacteria from leucina and valine, respectively, a high amount of these acid, mostly linked with over-fermentation Rottiers et al. (2019a). Concentrations of this compound exceeding $2 \%$ were found in cocoa from all districts, however, this concentration was reduced with roasting until reaching values below $1 \%$ (Figure 3 ). Some fine volatiles were assumed to be pulpderived (e.g. linalool, $\beta$-myrcene, 2-heptyl acetate) or intrinsic to the bean (e.g. 2heptanol, 2-heptanone, 2-pentanol) (Rottiers et al., 2019a). 2-heptanol (citrus, earth, fried, mushroom, oil) was found in considerable concentrations $(7 \%)$ in Nieva beans. Also, because Copallín and La Peca presented a significant interaction of roasting time and temperature, the optimization of the process allowed us to find the roasting parameters of $122.4^{\circ} \mathrm{C}$ for $35 \mathrm{~min}$ to obtain a maximized concentration of $3.91 \%$ of 2 heptanol in Copallín and $122.6^{\circ} \mathrm{C}$ for $20 \mathrm{~min}$ in La Peca to obtain a maximized concentration of $4.86 \%$ (Table 3 ).

The cocoa beans from Copallín and La Peca had 2,3,5-trimethylpyrazine concentrations that exceeded $5 \%$ in the most intense roasting conditions, therefore the production of 2,3,5-trimethylpyrazine by the roasting process is demonstrated, but it should also be noted that amounts of 2,3,5trimethylpyrazine less than $1 \%$ were produced in fermentation (Figure 3), in accordance with what was established by Hinneh et al. (2019) and Schwan and Wheals (2004). Likewise, the parameters necessary to maximize 2,3,5-trimethylpyrazine content up to $2.93 \%$ in Copallín were $122.4^{\circ} \mathrm{C}$ for 35 min, while in La Peca they were $122.6^{\circ} \mathrm{C}$ for 20 min to maximize up to $2.28 \%$.

Strecker aldehydes are formed during fermentation and roasting (Cordero et al.,
2019), benzeneacetaldehyde or phenylacetaldehyde (berry, geranium, honey, nut, pungent), were produced in small concentrations in the unroasted beans as a consequence of fermentation, these concentrations increased as roasting intensified; reaching significant values in the beans from Copallín, so the roasting parameters that maximized the concentration of benzeneacetaldehyde $(2.15 \%)$ were $122.4{ }^{\circ} \mathrm{C}$ and $\mathbf{3 5} \mathrm{min}$.

The high production of alcohols could be explained by fermentation of cocoa mucilaginous pulp sugars (Koné et al., 2016). Schwan and Wheals (2004) have reported 3methyl-1butanol, 2,3-butanediol and 2phenethyl alcohol as desirable for high quality cocoa products. The highest concentration of 2-phenethyl alcohol (fruit, honey, lilac, rose, wine) was found in Copallín, La Peca and Nieva, with concentrations exceeding $10 \%$. The highest concentrations of 2-phenethyl acetate (flower, honey, rose) had the unroasted beans of Copallín and La Peca, reaching 7\% (Figure 3 ). Acetoin could be produced by alcohol fermentation from piruvate, and butanodiol and it appears to be a precursor of tetramethylpyrazine (Rodriguez-Campos et al., 2011), then, this compound is desirable for flavor development in the fermentation process (UtrillaVázquez et al., 2020). Because it was not found in other references, we consider propyl acetate (celery, floral, pear, red fruit), acetoin acetate (fruit), and diethyl succinate (cotton, fabric, floral, fruit, wine) as the key aroma markers for APROCAM Criollo cocoa, acetoin acetate was found in all districts, in different concentrations. Nieva was the district with the highest concentration of propyl acetate, while Cajaruro had the highest concentration of acetoin acetate. Diethyl succinate was only found in Nieva and La Peca cocoa beans (Figure 3 ).

Table 3

Optimization parameters of the roasting process for the key aroma and technological markers found in APROCAM Criollo cocoa beans

\begin{tabular}{|c|c|c|c|c|c|}
\hline \multirow{2}{*}{ District } & \multirow{2}{*}{ Compound } & \multicolumn{2}{|c|}{ Parameters } & \multirow{2}{*}{$\begin{array}{c}\text { Optimal } \\
\text { value (\%) }\end{array}$} & \multirow{2}{*}{$\begin{array}{c}\text { Prediction } \\
\text { interval }\end{array}$} \\
\hline & & Time (min) & Temperature $\left({ }^{\circ} \mathrm{C}\right)$ & & \\
\hline Cajaruro & $\begin{array}{l}\text { Isovaleric acid } \\
\text { Acetic acid } \\
\text { 2,3,5-Trimethylpyrazine } \\
\text { 2,3-Dimethylpyrazine }\end{array}$ & 35 & 110 & $\begin{array}{l}0.00 \\
0.00 \\
2.92 \\
0.89 \\
\end{array}$ & $\begin{array}{r}0.00-2.02 \\
0.00-14.44 \\
2.22-3.62 \\
0.35-1.43 \\
\end{array}$ \\
\hline Copallín & $\begin{array}{l}\text { 2-heptanol } \\
\text { Benzeneacetaldehyde } \\
\text { 3-methylbutanal } \\
\text { 2,3,5-Trimethylpyrazine }\end{array}$ & 35 & 122.4 & $\begin{array}{l}3.91 \\
2.15 \\
6.54 \\
2.93 \\
\end{array}$ & $\begin{array}{l}2.87-4.94 \\
1.40-2.90 \\
4.26-8.81 \\
2.41-3.45 \\
\end{array}$ \\
\hline \multirow{3}{*}{ La Peca } & 2-heptanol & \multirow{3}{*}{20} & \multirow{3}{*}{122.6} & 4.86 & $2.58-7.13$ \\
\hline & 2-Phenylethyl acetate & & & 6.10 & $3.77-8.44$ \\
\hline & 2,3,5-Trimethylpyrazine & & & 2.28 & $1.99-2.58$ \\
\hline
\end{tabular}



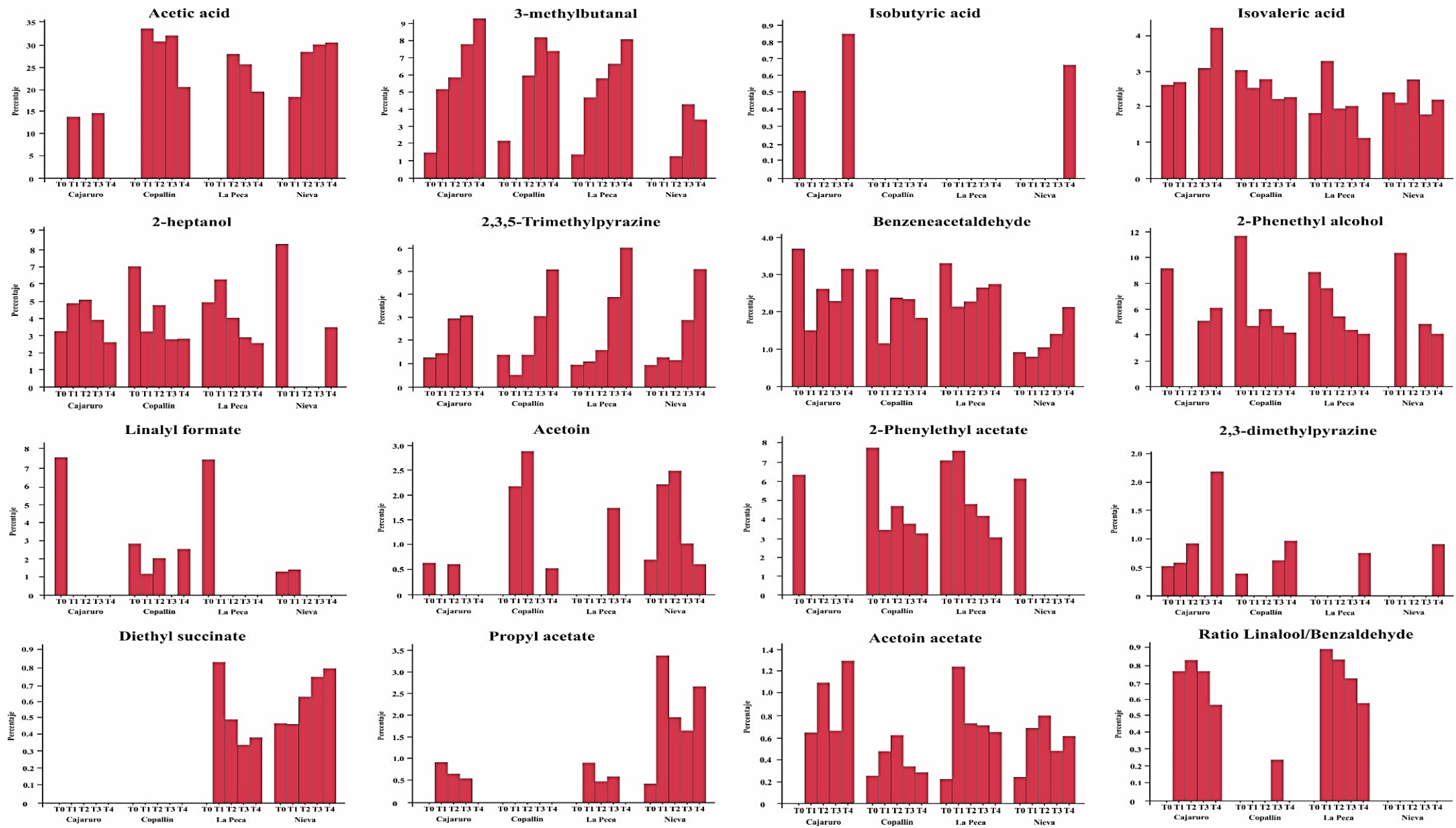

Figure 3. Key markers identified by HS-SPME GC-MS during the roasting process of Criollo cocoa beans. 
A

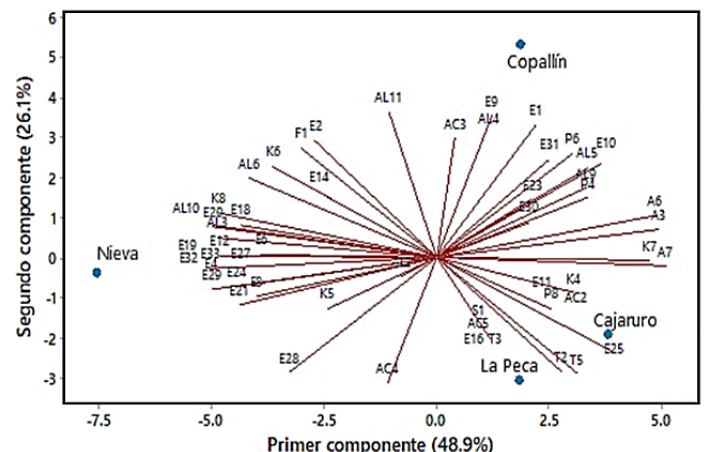

B

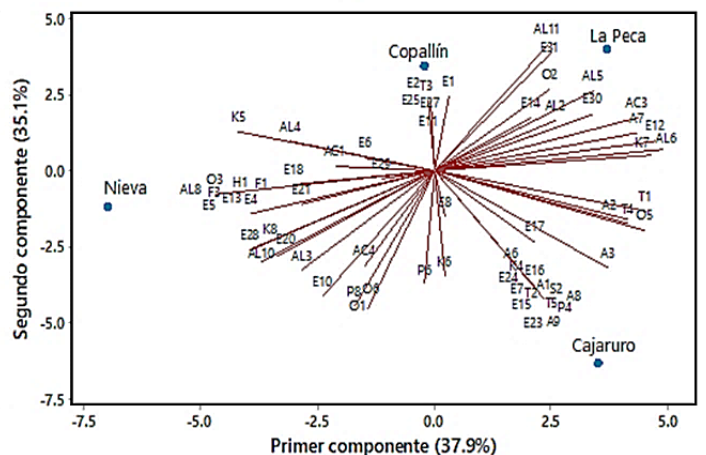

Figure 4. Principal components analysis of Criollo cocoa beans unroasted (A) and roasting for T1 (B).

Linalool (coriander, floral, lavender, lemon, rose) and benzaldehyde (sweet, bitter almond, cherry) are identified (Table 2). Linalool obviously proves to be an important key compound, which classifies the origins of cocoa. During the roasting process, the linalool content slightly decreases due to volatility but the relative difference between basic and flavour grade cocoas remains (Ziegleder, 1990). This component was identified mainly in roasted cocoa beans from Cajaruro and La Peca and its concentration decreased as there was a more intense roasting process (Figure 3 ). Ziegleder (1990) establishes the linalool/benzaldehyde ratio as an indicator of industrial quality control of unroasted cocoa, and a ratio of linalool/benzaldehyde higher than 0.3 indicates typical flavour grade cocoas and may be used as a flavour index. This ratio can only be observed in the cocoa beans from the districts of Cajaruro and La Peca, because linalool was produced only during roasting. The values of these ratios were between 0.56 and 0.82 for Cajaruro and between 0.57 and 0.89 for La Peca.

As reported for unroasted cocoa beans, the volatile fingerprint showed interesting potential for origin authentication of both unroasted and roasted cocoa beans (Marseglia et al., 2020). Therefore, the roasting treatments were subjected to a Principal Component Analysis (PCA) to find groups of districts formed according to the volatile fingerprint of the cocoa beans from each one. The PCA of the unroasted beans show a clear separation between districts, Figure 4A shows that the total variability of the samples was demonstrated in two PCs that explain $75 \%$ of the variance. It is shown that when the beans are not roasted, there is a clear separation between the Copallín and Nieva districts. This separation is caused by the greater number of esters that Copallin has, which are precisely those that give cocoa beans the fruity aroma. We can also note that La Peca and Cajaruro make up a group that distinguishes them from the other districts, mainly due to their content in pyrazines and terpenes. As the roasting process is carried out, the districts begin to form new groups; for example: in Figure 4B we can notice the presence of two PCs that explain $73 \%$ of the variance, so we can affirm that T1 (roasted at $110^{\circ} \mathrm{C}$ for $20 \mathrm{~min}$ ), causes Cajaruro and Copallín to form a group characterized mainly due to the presence of aldehydes, esters and linalool. This group differs considerably from Nieva, whose differences are characterized by pyrroles and esters (among them acetoin acetate); which in turn differs from La Peca. Another separation of groups can be noticed in the other districts (Figures S1, S2, S3 in supplementary).

\section{Conclusions}

APROCAM-Amazonas Criollo cocoa has a mainly fruit, floral and nutty sensory perception. It was possible to find new key aroma markers (propyl acetate, acetoin acetate, and diethyl succinate) for Criollo cocoa analyzed, which have not been identified in other Criollo or Trinitarian cocoas grown in other countries. The most abundant amount of volatile compounds related to the aroma of Criollo cocoa was found in roasted beans, since the roasting process caused the concentration and number of these compounds to decrease and allowed the generation of others such as pyrazines. The fingerprint of volatile compounds in Criollo cocoa changed according to the roasting parameters, finding that these parameters allow the formation of different groups between the districts where it is grown; therefore, although the cocoa belongs to the same production region, there is variability in its volatile fingerprint that is specific to each geographical location. For this reason, we recommended that to optimize the roasting process, the origin of each cocoa bean must be taken into account, and the appropriate 
parameters must be used in order to preserve the aroma of the bean and make chocolates with aromatic profiles according to origin. This should be considered mainly in the manufacture of fine chocolates to maintain the "Terroir" of Peruvian fine chocolate.

\section{Acknowledgments}

A special acknowledgement to Multi-Service Cooperative APROCAM for the permission for the use of their facilities. This research was funded by Project $N^{\circ} 16808-2016 /$ Proyecto-Cacao, of the Programa Nacional de Innovación Agraria (PNIA) of the Instituto Nacional de Innovación Agraria of the Peruvian Government, the Universidad Nacional Toribio Rodríguez de Mendoza de Amazonas and the Pontificia Universidad Católica del Perú.

\section{ORCID}

E.M. Castro-Alayo (1) https://orcid.org/0000-0003-4322-8980 M.G. Valle-Epquín (D) https://orcid.org/0000-0003-0060-2231 C.R. Balcázar-Zumaeta ${ }^{\mathbb{D}}$ https://orcid.org/0000-0002-3033-6440 E.A. Auquiñivín-Silva (1) https://orcid.org/0000-0002-9226-9896 A.B. Fernández-Jeri $(\mathbb{D}$ https://orcid.org/0000-0001-9476-1078 G. Idrogo-Vásquez (D) https://orcid.org/0000-0003-1044-5006

\section{References}

Aculey, P.C.; Snitkjaer, P.; Owusu, M.; et al. 2010. Ghanaian Cocoa Bean Fermentation Characterized by Spectroscopic and Chromatographic Methods and Chemometrics. J. Food Sci. 75: S300-S307.

Afoakwa, E.O., 2010. Chocolate science and technology, First edition. ed. Wiley-Blackwell. Chichester, U.K., Chichester, U.K.

Afoakwa, E.O.; Paterson, A.; Fowler, M.; et al. 2008. Flavor Formation and Character in Cocoa and Chocolate: A Critical Review. Crit. Rev. Food Sci. Nutr. 48: 840-857.

Ahmed, S.; Rattanpal, H.S.; Gul, K.; et al. 2019. Chemical composition, antioxidant activity and GC-MS analysis of juice and peel oil of grapefruit varieties cultivated in India. J. Integr. Agric. 18: 1634-1642.

Álvarez, C.; Pérez, E.; Lares, M.D.C.; et al. 2016. Identification of the volatile compounds in the roasting Venezuela criollo cocoa beans by gas chromatography- spectrometry mass. J Nutr Health Food Eng. 5(4): 659-666.

Aprotosoaie, A.C.; Luca, S.V.; Miron, A. 2016. Flavor Chemistry of Cocoa and Cocoa Products - An Overview. Compr. Rev. Food Sci. Food Saf. 15: 7391.

Ascrizzi, R.; Flamini, G.; Tessieri, C.; et al. 2017. From the raw seed to chocolate: Volatile profile of Blanco de Criollo in different phases of the processing chain. Microchem. J. 133: 474-479.

Braga, S.C.G.N.; Oliveira, L.F.; Hashimoto, J.C.; et al. 2018. Study of volatile profile in cocoa nibs, cocoa liquor and chocolate on production process using GC × GC-QMS. Microchem. J. 141: 353-361.

Caligiani, A.; Palla, L.; Acquotti, D.; et al. 2014. Application of $1 \mathrm{H}$ NMR for the characterisation of cocoa beans of different geographical origins and fermentation levels. Food Chem. 157: 94-99.

Castro-Alayo, E.M.; Idrogo-Vásquez, G.; Siche, R.; et al. 2019. Formation of aromatic compounds precursors during fermentation of Criollo and Forastero cocoa. Heliyon 5: e01157.

Cordero, C.; Guglielmetti, A.; Sgorbini, B.; et al. 2019. Odorants quantitation in high-quality cocoa by multiple headspace solid phase micro-extraction: Adoption of FID-predicted response factors to extend method capabilities and information potential. Anal. Chim. Acta 1052: 190-201.

Counet, C.; Callemien, D.; Ouwerx, C.; et al. 2002. Use of Gas Chromatography-OIfactometry To Identify Key Odorant Compounds in Dark Chocolate. Comparison of Samples before and after Conching. J. Agric. Food Chem. 50: 2385-2391.

De Brito, E.S.; Garcia, P.; Cortelazzo, M.G.A.L. 2000. Structural and chemical changes in cocoa (Theobroma cacao L) during fermentation. J. Sci. Food Agric. 88: 281-288.

Di Carro, M.; Ardini, F.; Magi, E. 2015. Multivariate optimization of headspace solid-phase microextraction followed by gas chromatography-mass spectrometry for the determination of methylpyrazines in cocoa liquors. Microchem. J. 121: 172177.

Djikeng, F.T.; Teyomnou, W.T.; Tenyang, N.; et al. 2018. Effect of traditional and oven roasting on the physicochemical properties of fermented cocoa beans. Heliyon 4: e00533.

El Atki, Y.; Aouam, I.; El Kamari, F.; et al. 2020. Phytochemistry, antioxidant and antibacterial activities of two Moroccan Teucrium polium L. subspecies: Preventive approach against nosocomial infections. Arab. J. Chem. 13: 38663874.

FEMA. 2018. Flavor Ingredient Library. Available in: https://www.femaflavor.org/about

Fernández-Romero, E.; Chavez-Quintana, S.G.; Siche, R.; et al. 2020. The Kinetics of Total Phenolic Content and Monomeric Flavan-3-ols during the Roasting Process of Criollo Cocoa. Antioxidants 9: 146.

Frauendorfer, F.; Schieberle, P. 2008. Changes in Key Aroma Compounds of Criollo Cocoa Beans During Roasting. J. Agric. Food Chem. 56: 10244-10251.

Frauendorfer, F.; Schieberle, P. 2006. Identification of the Key Aroma Compounds in Cocoa Powder Based on Molecular Sensory Correlations. J. Agric. Food Chem. 54: 5521-5529.

García-Alamilla, P.; Lagunes-Gálvez, L.M.; BarajasFernández, J.; et al. 2017. Physicochemical Changes of Cocoa Beans during Roasting Process. J. Food Qual. 2017: 2969324.

Hinneh, M.; Van de Walle, D.; Tzompa-Sosa, D.A.; et al. 2019. Tuning the aroma profiles of Forastero cocoa liquors by varying pod storage and bean roasting temperature. Food Res. Int. 125: 108550.

ICCO. 2017. Fine or Flavour Cocoa. Available in: https://www.icco.org/about-cocoa/fine-or-flavourcocoa.html

loannone, F.; Di Mattia, C.D.; De Gregorio, M.; et al. 2015. Flavanols, proanthocyanidins and antioxidant activity changes during cocoa (Theobroma cacao L.) roasting as affected by temperature and time of processing. Food Chem. 174: 256-262.

Jinap, S.; Rosli, W.; Russly, A.R.; et al. 1998. Effect of Roasting Time and Temperature on Volatile Component Profiles during Nib Roasting of Cocoa Beans (Theobroma cacao). J. Sci. Food Agric. 77: 441-448.

Koné, M.K.; Guéhi, S.T.; Durand, N.; et al. 2016. Contribution of predominant yeasts to the occurrence of aroma compounds during cocoa bean fermentation. Food Res. Int. 89: 910-917.

Kongor, J.E.; Hinneh, M.; de Walle, D.V.; et al. 2016. Factors influencing quality variation in cocoa (Theobroma cacao) bean flavour profile-A review. Food Res. Int. 82: 44-52.

Marseglia, A.; Musci, M.; Rinaldi, M.; et al. 2020. Volatile fingerprint of unroasted and roasted cocoa beans (Theobroma cacao L.) from different geographical origins. Food Res. Int. 132: 109101.

Qin, X.-W.; Lai, J.-X.; Tan, L.-H.; et al. 2016 Characterization of volatile compounds in Criollo, Forastero, and Trinitario cocoa seeds (Theobroma cacao L.) in China. Int. J. Food Prop. 20: 2261-2275.

Rodriguez-Campos, J.; Escalona-Buendía, H.B.; Contreras-Ramos, S.M.; et al. 2012. Effect of 
fermentation time and drying temperature on volatile compounds in cocoa. Food Chem. 132: 277-288.

Rodriguez-Campos, J.; Escalona-Buendía, H.B.; OrozcoAvila, I.; et al. 2011. Dynamics of volatile and nonvolatile compounds in cocoa (Theobroma cacao L.) during fermentation and drying processes using principal components analysis. Food Res. Int. 44: 250-258.

Rottiers, H.; Tzompa Sosa, D.A.; De Winne, A.; et al. 2019a. Dynamics of volatile compounds and flavor precursors during spontaneous fermentation of fine flavor Trinitario cocoa beans. Eur. Food Res. Technol. 245: 1917-1937.

Rottiers, H.; Tzompa Sosa, D.A.; Van de Vyver, L.; et al. 2019b. Discrimination of Cocoa Liquors Based on Their Odor Fingerprint: a Fast GC Electronic Nose Suitability Study. Food Anal. Methods 12: 475-488.

Schwan, R.F.; Wheals, A.E. 2004. The Microbiology of Cocoa Fermentation and its Role in Chocolate Quality. Crit. Rev. Food Sci. Nutr. 44: 205-221.

Sukha, D.A.; Umaharan, P.; Butler, D.R. 2017. Evidence for applying the concept of "Terroir" in cocoa (Theobroma cacao L.) flavour and quality attributes. Presented at the International Symposium on Cocoa Research (ISCR), International Cocoa Organization, Lima, Peru.

Taş, N.G.; Gökmen, V. 2016. Effect of alkalization on the Maillard reaction products formed in cocoa during roasting. Food Res. Int. 89: 930-936.
Tran, P.D.; Van de Walle, D.; De Clercq, N.; et al. 2015. Assessing cocoa aroma quality by multiple analytical approaches. Food Res. Int. 77: 657-669.

Tuenter, E.; Delbaere, C.; De Winne, A.; et al. 2020. Nonvolatile and volatile composition of West African bulk and Ecuadorian fine-flavor cocoa liquor and chocolate. Food Res. Int. 130: 108943.

Utrilla-Vázquez, M.; Rodríguez-Campos, J.; AvendañoArazate, C.H.; et al. 2020. Analysis of volatile compounds of five varieties of Maya cocoa during fermentation and drying processes by Venn diagram and PCA. Food Res. Int. 129: 108834.

Van Durme, J.; Ingels, I.; De Winne, A. 2016. Inline roasting hyphenated with gas chromatographymass spectrometry as an innovative approach for assessment of cocoa fermentation quality and aroma formation potential. Food Chem. 205: 66-72.

Ziegleder, G. 2009. Flavour Development in Cocoa and Chocolate, in: Industrial Chocolate. Manufacture and Use. Blackwell Publishing. York, UK, pp. 169-191.

Ziegleder, G. 1990. Linalool contents as characteristic of some flavor grade cocoas. Z. Für Leb. -Forsch. A 191: 306-309.

Zzaman, W.; Bhat, R.; Yang, T.A. 2014. Application of Response Surface Methodology to Optimize Roasting Conditions in Cocoa Beans Subjected to Superheated Steam Treatments in Relevance to Antioxidant Compounds and Activities. Dry. Technol. 32: 1104-1111. 